\title{
Analysis of the diagnostic value of CD138 for chronic endometritis, the risk factors for the pathogenesis of chronic endometritis and the effect of chronic endometritis on pregnancy: a cohort study
}

Yu-qing Chen ${ }^{1 *}$, Rui-li Fang ${ }^{2}$, Yuan-na Luo ${ }^{3}$ and Can-qiao Luo ${ }^{4}$

\begin{abstract}
Background: To investigate the role of CD138 immunohistochemistry in the diagnosis of chronic endometritis (CE) and the risk factors for assisted conception patients having CE complications.

Methods: Ninety-three patients, with normal uterine shape confirmed by examination and who were planning to undergo assisted conception treatments, were selected as research subjects. Endometrial tissue was isolated for routine hematoxylin and eosin (HE) and CD138 immunohistochemical staining. Additionally, the disease histories of patients were collected, and the reproductive prognosis was followed up.

Results: (1) CE detection rate: The rate of CD138 immunohistochemical staining was greater than that of HE staining (27.96 \% vs. $26.89 \%, P<0.05)$; (2) Pregnancy rate: the pregnancy rate of CD138-positive patients (7.7 \%) was lower than the pregnancy rate of CD138-negative patients $(31.3 \%)(p=0.017<0.05)$; (3) The results from univariate analysis showed that a previous history of prolonged menstrual bleeding episodes, an abortion history, and complications of fallopian tube obstruction were associated with CE $(P<0.05)$. The results of logistic regression analysis confirmed that prolonged menstrual bleeding episodes $(P=0.014, \mathrm{OR}=5.394,95 \% \mathrm{Cl} 1.405-20.699)$, a previous abortion history $(P=0.029, \mathrm{OR}=3.194,95 \% \mathrm{Cl} 1.125-9.073)$, and fallopian tube obstruction $(P=0.028$, $\mathrm{OR}=3.274,95 \% \mathrm{Cl}$ 1.139-9.415) were independent risk factors for positive CD138 results.

Conclusions: CD138 immunohistochemistry can improve the CE diagnosis rate. A previous history of prolonged menstrual bleeding episodes, an abortion history, and a history of fallopian tube obstruction are risk factors for chronic endometritis, and a CD138 immunohistochemical examination should be advised among them.
\end{abstract}

Keywords: Chronic endometritis, CD138, Infertility

Abbreviations: CD138, the transmembrane heparin sulfate proteoglycan syndecan-1; CE, Chronic endometritis; $\mathrm{HE}$, Hematoxylin and eosin

\footnotetext{
* Correspondence: fangchenyq@163.com

${ }^{1}$ Department of Gynecology and Obstetrics, First Affiliated Hospital, Sun

Yat-sen University, Guangzhou, Guangdong Province, People's Republic of

China

Full list of author information is available at the end of the article
} 


\section{Background}

Infertility is a relatively common gynecological disease whose incidence has increased in recent years. Relevant studies have shown that the incidence of chronic endometritis $(\mathrm{CE})$ in infertile patients ranges from $0.2-46 \%[1,2]$. Infertility and natural abortion may be associated with and CE $[1,3,4]$. Recent studies pointed out that the prevalence of $\mathrm{CE}$ was $14 \%$ in patients with recurrent implantation failure (RIF) after IVF [5] and women with CE had lower implantation rates $(11.5 \%)$ in the IVF cycle following treatment than did those with CE absent (32.7 \%) [6]. Furthermore, Cicinelli et al. demonstrated that CE is a common finding in women complaining of repeated implantation failure and appropriate antibiotic treatment had improved significantly the rate of successful pregnancies in women without signs of CE compared with women who were with persistent CE [7]. The reports suggest the presence of CE may alter endometrial receptivity and negatively affect fertility outcome.

However, CE normally does not show symptoms and is easily overlooked in clinical practice $[2,4,8]$. The diagnosis of $\mathrm{CE}$ is based on the detection of abnormal plasma cell infiltration in the endometrial stroma [9] (Kasius et al., 2011). Using conventional hematoxylin and eosin (HE) staining, it is sometimes difficult to distinguish the plasma cells from the fibroblasts and monocytes of the endometrial stroma; thus, the success rate of accurate diagnosis and treatment of $\mathrm{CE}$ is not high $[9,10]$. The transmembrane heparin sulfate proteoglycan syndecan-1 (CD138) is a syndecan, which is a specific marker of plasma cells. Therefore, in current clinical practice, CD138 immunohistochemistry is used for the detection of chronic endometritis to improve its diagnosis rate [11]. However, because CE tends to be overlooked in clinical practice, and clinicians have a limited understanding of the association between $\mathrm{CE}$ and infertility, this method is rarely used for further examination. The present study has compared the detection rate of chronic endometritis by HE and CD138 immunohistochemical staining and investigated the difference in sensitivity between CD138 immunohistochemical staining and conventional HE staining in the diagnosis of chronic endometritis, while further analyzing the relevant influential factors for the pathogenesis of chronic endometritis to provide a reference for clinical treatment.

\section{Methods}

\section{General information}

We prospectively collected information from 93 patients who recently had a laparoscopy and hysteroscopy examination and planned to undergo assisted conception treatment at the First Affiliated Hospital of Sun Yat-sen University between April 2013 and December 2013 due to infertility. The ages of the patients were between 23 and 43 years, with an average age of $34.36 \pm 4.63$ years. The average time of pregnancy was $1.3 \pm 1.3$, and the average time of giving birth was $0.50 \pm 0.56$. None of the 93 assisted conception patients had chronic abdominal pain or lumbosacral pain. Laparoscopy examinations confirmed that 43 patients had unilateral fallopian tube obstruction, and one patient had bilateral fallopian tube obstruction. Of these patients, 12 patients had prolonged menstrual bleeding episodes, 32 patients had undergone abortion surgery, 12 patients had a pelvic inflammatory disease history, 6 patients had history of cervical mycoplasma infection, 6 patients had history of cervical chlamydial infection and 12 patients had complications related to low sperm counts in male partners. A prolonged menstrual bleeding episode refers to a prolonged menstrual period or bleeding between menstrual cycles.

The case inclusion criteria consisted or patients who (1) were married, (2) had a regular sexual life for longer than one year without contraception that did not result in pregnancy, (3) were clinically diagnosed with fallopian tube-factor infertility, male factor infertility, or infertility for an unknown reason.

Case exclusion criteria included patients who had (1) complications with other intrauterine lesions, such as intrauterine adhesions, submucosal uterine fibroids, uterine septum, and other uterine malformations; (2) complications of uterine fibroids, endometriosis, ovarian tumor, or hydrosalpinx; (3) female hormone abnormalities; (4) vaginitis found by vaginal leucorrhea examination (including bacterial vaginosis or vulvovaginal yeast vaginosis or trichomonas vaginitis), pelvic tenderness during gynecological examination, or suspected vaginitis or pelvic inflammatory disease or acute infection period.

The present study has been reviewed and approved by the Ethics Committee ([2014] 64) of the First Affiliated Hospital of Sun Yat-sen University, and an informed consent was signed on by the patients involved.

\section{Specimen collection}

All patients underwent laparoscopic surgery during the early follicular phase. After anesthesia, an endometrium biopsy was collected before the hysteroscopic and laparoscopic surgery was commenced.

\section{Processing of the pathological specimens of the endometrium}

After the tissue was collected, it was immediately immersed in $10 \%$ neutral formaldehyde for fixation. After ethanol gradient dehydration, the tissue was cleared with xylene. The tissue was immersed in paraffin for embedding, and $4 \mu \mathrm{m}$ serial sections were cut. After HE staining, immunohistochemical staining was performed. Immunohistochemistry was performed by following the detailed procedures of the kit (DAKO, Carpinteria, CA, USA). 
The pathological results were independently analyzed by two experienced pathologists to detect plasma cells for diagnosis. In the case of a disagreement, the obtained results were discussed by the two pathologists. If necessary, a third pathologist was consulted to resolve a dispute.

Pathological diagnostic criteria for chronic endometritis [9, 12]: For HE-stained specimens, the criteria consisted of the following: (1) For the diagnosed chronic endometritis group, typical plasma cells were visible in the endometrial stroma. (2) For the suspected chronic endometritis group, no plasma cells were detected in the HE-stained endometrium, and endometrial stromal cells showed spindle-like shapes similar to fibrosis and lymphocytes that were clustered focally in endometrial stromal cells (Fig. 1a). (3) For the non-chronic endometritis group, neither of the first two conditions were met in HE-stained specimens (Fig. 1b).

The criteria for CD138 immunohistochemically stained specimens [9, 12]: After CD138 immunohistochemistry staining, the plasma cell membrane showed strong positive staining, while the cytoplasm showed weak positive staining. Under microscopic observation, the
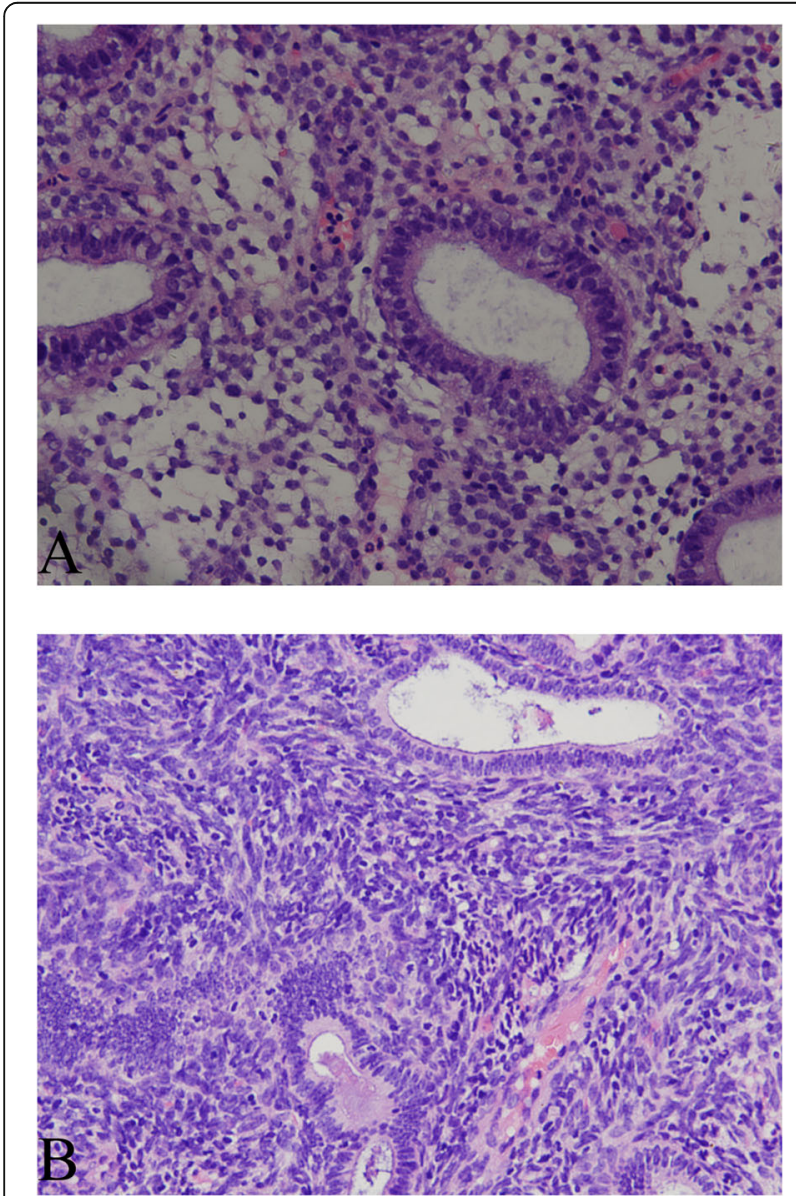

Fig. 1 HE staining performance of suspected chronic endometritis (a) and non-chronic endometritis (b) $(H E, \times 200)$ nucleus was rounded but located on one side of the cell, and the thick chromatin was radially arranged along the nuclear membrane to form a wheel shape (Fig. 2a). The cytoplasm was basophilic. (1) For specimens diagnosed as chronic endometritis, in each $400 \mathrm{x}$ magnification field, five or more typical plasma cells were observed in the endometrial stroma. (2) For specimens not diagnosed as chronic endometritis, no plasma cells were observed in CD138 immunohistochemically stained specimens, or less than five plasma cells were observed in each 400x magnification field (Fig. 2b).

\section{Follow-up examinations}

All patients underwent at least 12 months of follow-up after the operation; the interval of follow-up evaluations was three months. The information needed to calculate the cumulative pregnancy rate of all patients was collected through phone calls and outpatient visits.

\section{Statistical analysis}

All data were analyzed with the SPSS 13.0 statistical software package. Measured data are represented by means \pm
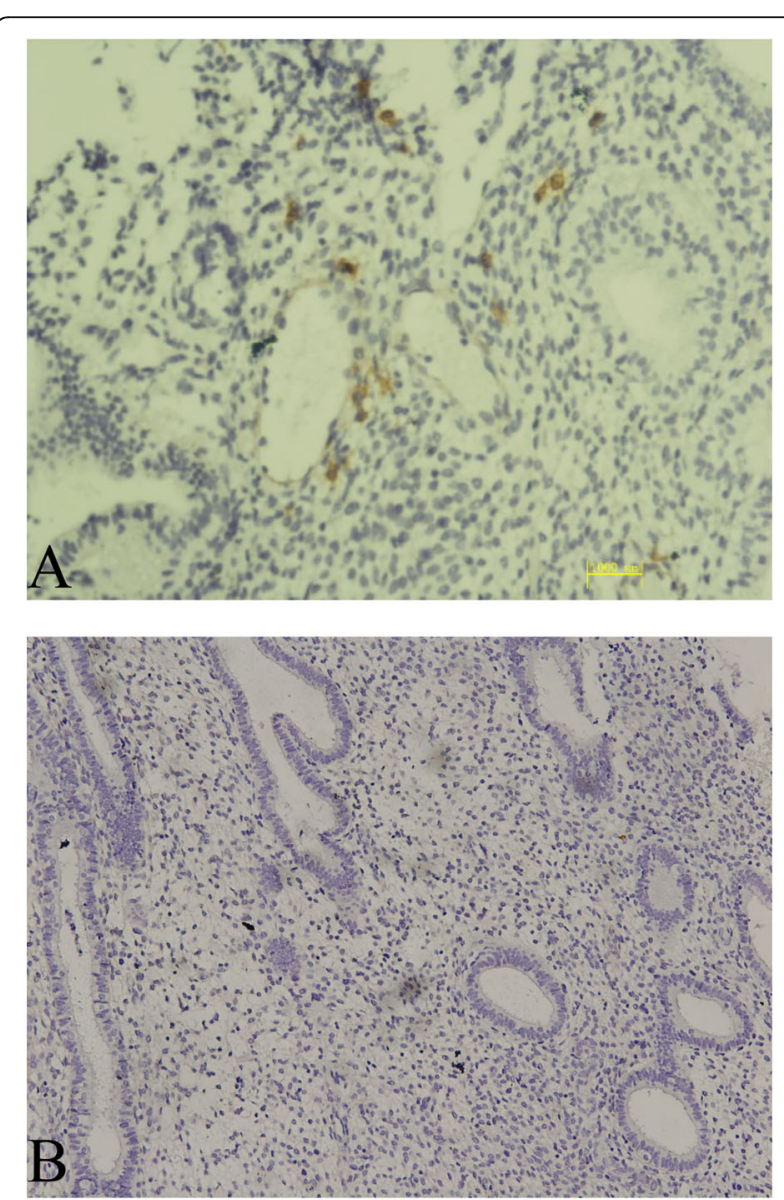

Fig. 2 Positive staining of CD138 in chronic endometritis (a) and negative staining of CD138 in non-chronic endometritis (b) 
standard deviations $(x \pm s)$. If the data of two samples were normally distributed and the two population variances were equal at the significant level, two independent samples $t$ test analysis was used. For data that did not have a normal distribution or were not equal at the significant level, a Wilcoxon rank-sum test was used. A Chi-square test was used for the cell count data. For data that did not meet the requirement for a Chi-square test, Fisher's exact test was used. Pearson X2 independence test was used for the association analysis of categorical variables. All variables with a value of $p<0.05$ in the univariate analysis were included in the multivariate unconditional logistic regression model by the stepwise forward method. A value of $P<0.05$ indicated statistical significance.

\section{Results}

The difference in the positive staining rate between the HE group and the CD138 immunohistochemistry staining group A Chi-square test was used to compare the positive rate of the two groups (Table 1). The positive rate of the HE group was 0 , and the suspected positive rate was $26.89 \%$ $(25 / 93)$. Both rates were lower than the positive rate of the CD138 immunohistochemistry group (27.96 \%, 26 / 93), and the difference was statistically significant $(P=0.041)$. According to CD138 immunohistochemical results, the 93 patients were divided into two groups, the chronic endometritis group ( $\mathrm{CE}, n=26)$ and the non-chronic endometritis group (NCE, $n=67$ ). The clinical data of the two groups are shown in Table 2. Except for a significant difference in oviduct blockage between the two groups $(P=0.030)$, the differences of all other variables were not statistically significant $(P>0.05)$.

The effect of negative and positive CD138 results on the implantation of fertilized eggs in infertile patients

All 93 patients underwent assisted pregnancy treatment at an opportune time after surgery (IVF-ET and five cases of artificial insemination). And the five patients were all from the non-chronic endometritis group. Twenty-three patients successfully became pregnant after the operation, 20 patients underwent IVF-ET assisted procedures, and three patients received artificial insemination. The pregnancy rate of CD138 negative patients $(31.3 \%, 21 / 67)$ was higher than that of CD138 positive patients $(7.7 \%, 2 / 26)$,

Table 1 The comparison of diagnosis rate for CE between HE and immunohistochemical staining of CD138

\begin{tabular}{llllll}
\hline & & \multicolumn{2}{l}{ CD138 } & & \\
\cline { 3 - 4 } & & Positive & Negative & Totality & \\
\hline HE & Positive & 0 & 0 & 0 & \\
& Probable positive & 3 & 22 & 25 & $P=0.041$ \\
Negative & 23 & 45 & 68 & \\
totality & 26 & 67 & 93 & \\
\hline
\end{tabular}

Table 2 The comparison of the general conditions

\begin{tabular}{|c|c|c|c|c|}
\hline & $\begin{array}{l}\text { CE } \\
(n=26)\end{array}$ & $\begin{array}{l}\text { NCE } \\
(n=67)\end{array}$ & $t / X^{2} / Z$ & $P$ \\
\hline Age $(y)^{1)}$ & $35.50 \pm 3.89$ & $33.92 \pm 4.84$ & -1.515 & 0.130 \\
\hline $\begin{array}{l}\text { Body mass } \\
\text { index }\left(\mathrm{kg} / \mathrm{m}^{2}\right)^{1)}\end{array}$ & $21.50 \pm 1.54$ & $21.31 \pm 3.42$ & -0.767 & 0.443 \\
\hline $\begin{array}{l}\text { Menstrual } \\
\text { Cycle (day) }\end{array}$ & $28.36 \pm 2.24$ & $29.40 \pm 3.93$ & -0.666 & 0.505 \\
\hline $\begin{array}{l}\text { menstrual } \\
\text { period }(\text { day })^{1)}\end{array}$ & $5.57 \pm 1.28$ & $5.22 \pm 1.70$ & -1.007 & 0.314 \\
\hline Gravidity $(n)^{1)}$ & $1.29 \pm 1.18$ & $1.26 \pm 1.36$ & -0.276 & 0.782 \\
\hline Parity $(n)^{1)}$ & $0.38 \pm 0.48$ & $0.54 \pm 0.58$ & -1.104 & 0.270 \\
\hline $\begin{array}{l}\text { infertility } \\
\text { duration }(y)^{1)}\end{array}$ & $3.42 \pm 1.47$ & $3.33 \pm 1.31$ & -0.630 & 0.529 \\
\hline $\begin{array}{l}\text { Primary } \\
\text { infertility }(n)^{2)}\end{array}$ & $9(34.6)$ & $28(41.8)$ & 0.403 & 0.526 \\
\hline $\begin{array}{l}\text { male asthenospermia } \\
\text { and oligospermia }(n)^{2)}\end{array}$ & $6(23.1)$ & $6(9.0)$ & 3.324 & 0.068 \\
\hline Tubal blockage $(n)^{2)}$ & $17(65.4)$ & $27(40.3)$ & 4.729 & $0.030^{*}$ \\
\hline
\end{tabular}

1. CE: Patients with chronic endometritis; NCE: patients without chronic endometritis

2. ${ }^{1)}$ Mann-Whitney u Test ${ }^{2)} \mathrm{Chi}$-square test

3. Significant difference at $p<0.05$

and the difference was statistically significant $(P=0.017)$. The results are shown in Table 3 . In addition, we independently evaluated the pregnancy rate of IVF cycles and the results were significantly different $(P=0.048)$.

\section{Analysis of CD138 positive risk factors}

The clinical data of CD138 positive patients were retrospectively analyzed. Pearson's $X^{2}$ independence test was applied to each possible factor of CD138 positive patients to perform an association analysis of categorical variables (Table 3). The results showed that prolonged menstrual bleeding episodes $\left(\chi^{2}=10.250, P<0.05, r=0.315\right)$, a previous abortion history $\left(\chi^{2}=6.042, P<0.05, \quad r=0.247\right)$, fallopian tube obstruction $\left(\chi^{2}=4.729, P<0.05, r=0.220\right)$ were associated with $\mathrm{CE}$. Moreover, these factors were used as independent variables, and CD138 was used as the dependent variable in multivariate logistic regression analysis. The results confirmed that prolonged menstrual bleeding episodes $(P=0.014, O R=5.394,95 \% C I 1.405$ 20.699), a previous abortion history $(P=0.029, O R=3.194$, $95 \% C I$ 1.125-9.073), and fallopian tube obstruction $(P=0.028, O R=3.274,95 \% C I 1.139-9.415)$ are independent risk factors of positive CD138 results (Table 4).

\section{Discussion}

Infertility is a common gynecological disease. The endometrium is the location for fertilized egg implantation, and chronic endometritis may cause damage to endometrial receptivity, resulting in infertility or miscarriage. Relevant studies [4] have shown that the implantation rate of embryo transfer in infertile patients complicated 
Table 3 The related risk factors for chronic endometritis

\begin{tabular}{|c|c|c|c|c|c|c|}
\hline factors & $\begin{array}{l}\text { Totality } \\
\mathrm{n}\end{array}$ & $\begin{array}{l}\text { CE } \\
(n=26)\end{array}$ & $\begin{array}{l}\text { NCE } \\
(n=67)\end{array}$ & $x 2$ & $P$ & $r$ \\
\hline \multicolumn{7}{|c|}{$\begin{array}{l}\text { prolonged menstrual } \\
\text { bleeding }\end{array}$} \\
\hline Yes & 12 & 8 & 4 & 10.25 & $0.003^{*}$ & 0.315 \\
\hline No & 81 & 18 & 63 & & & \\
\hline \multicolumn{7}{|l|}{ delivery history } \\
\hline Yes & 42 & 9 & 33 & 1.621 & 0.203 & 0.131 \\
\hline No & 51 & 17 & 34 & & & \\
\hline \multicolumn{7}{|c|}{$\begin{array}{l}\text { previous abortion } \\
\text { history }\end{array}$} \\
\hline Yes & 32 & 14 & 18 & 6.042 & $0.014^{*}$ & 0.247 \\
\hline No & 61 & 12 & 49 & & & \\
\hline \multicolumn{7}{|c|}{$\begin{array}{l}\text { history of spontaneous } \\
\text { abortion }\end{array}$} \\
\hline Yes & 14 & 6 & 8 & 1.817 & 0.178 & 0.138 \\
\hline No & 79 & 20 & 59 & & & \\
\hline \multicolumn{7}{|c|}{ history of vaginitis } \\
\hline Yes & 51 & 15 & 36 & 0.119 & 0.73 & 0.036 \\
\hline No & 42 & 11 & 31 & & & \\
\hline \multicolumn{7}{|l|}{$\begin{array}{l}\text { history of pelvic } \\
\text { inflammation }\end{array}$} \\
\hline Yes & 12 & 6 & 6 & 3.324 & 0.068 & 0.186 \\
\hline No & 81 & 20 & 61 & & & \\
\hline \multicolumn{7}{|c|}{$\begin{array}{l}\text { History of cervical } \\
\text { mycoplasma infection }\end{array}$} \\
\hline Yes & 6 & 3 & 3 & 1.547 & 0.214 & 0.128 \\
\hline No & 87 & 23 & 64 & & & \\
\hline \multicolumn{7}{|c|}{$\begin{array}{l}\text { History of cervical } \\
\text { chlamydial infection }\end{array}$} \\
\hline Yes & 6 & 3 & 3 & 1.547 & 0.214 & 0.128 \\
\hline No & 87 & 23 & 64 & & & \\
\hline \multicolumn{7}{|l|}{ Tubal blockage } \\
\hline Yes & 44 & 17 & 27 & 4.729 & $0.030^{*}$ & 0.22 \\
\hline No & 49 & 9 & 40 & & & \\
\hline \multicolumn{7}{|l|}{$\begin{array}{l}\text { pregnancy } \\
\text { outcome }\end{array}$} \\
\hline pregnancy & 23 & 2 & 21 & 5.629 & $0.017^{*}$ & -0.246 \\
\hline unpregnancy & 70 & 24 & 46 & & & \\
\hline
\end{tabular}

*Significant difference at $p<0.05$ with chronic endometritis is significantly lower than that of infertile patients without endometritis. Erika B et al. [5] also found a higher failure rate of implantation into the endometrium in patients with chronic endometritis. The preventive use of antibiotics in patients with repeated failure of implantation and recurrent miscarriages can effectively improve their reproduction prognosis $[4,5]$. Similarly, Cicinelli E et al. further confirmed the results in the recent study [7]. If the chronic endometritis is not treated, the success rate of natural conception and artificial insemination will be reduced, and these processes may even result in adverse obstetric complications [13]. Kotaro Kitaya et al. [4] analyzed and labeled syndecan-1 via immunohistochemical methods to identify stromal plasma cells. These researchers determined that among patients with habitual abortion, approximately $10 \%$ of the patients suffered from chronic endometritis, while in habitual abortion patients without a known cause, the prevalence of endometritis was approximately 12-13\%. All patients with a chronic endometritis disease history had a miscarriage history during the first three months of pregnancy. This finding indicates that chronic endometritis is a factor for habitual abortion that should not be ignored. The results from the present study have shown that among infertile patients, the detection rate of chronic endometritis is $27.96 \%$. Moreover, among patients who received assisted reproductive technology, the cumulative pregnancy rate within one year after surgery in the nonchronic endometritis group $(31.3 \%)$ was significantly higher than that of the endometritis group (7.7 \%) $(P=0.017<0.05)$, indicating that chronic endometritis may be one of the causes of infertility in patients. Improvement of the inflammation status of the endometrium, such as antibiotic treatment can improve the pregnancy rate in patients.

Chronic endometritis normally does not show symptoms. As in the 26 chronic endometritis patients diagnosed in our study, these patients do not experience abdominal or lumbosacral pain or symptoms of pelvic inflammatory disease, and their diagnosis mainly relies on the existence of plasma cells. However, because the uterine body is isolated from the immune system, the immune response of the endometrium is not strong. A low number of plasma cells in the lesion or an improperly processed specimen and interference from the granulosa cells and fibroblasts in the tissue will make it

Table 4 Logistic regression analysis for chronic endometritis

\begin{tabular}{lcccc}
\hline Variable & $B$ value & $P$ value & Odds ratio & $95 \% \mathrm{Cl}$ \\
\hline prolonged menstrual bleeding & 1.685 & $0.014^{*}$ & 5.394 & $1.405-20.699$ \\
previous abortion history & 1.161 & $0.029^{*}$ & 3.194 & $1.125-9.073$ \\
Tubal blockage & 1.186 & $0.028^{*}$ & 3.274 & $1.139-9.415$ \\
\hline
\end{tabular}

*Significant difference at $p<0.05$ 
difficult to identify the plasma cells in the HE-stained sections. Even a highly experienced pathologist may miss the diagnosis. In addition, the histological changes in the endometrium at different stages can also interfere with the identification of plasma cells and the diagnosis of CE. During late menstruation or early endometrium proliferation, significant mononuclear cell infiltration, active mitosis in the stroma, stroma cell proliferation, or plasma cell-like stroma cell infiltration can occur, and significant premenstrual reactions occur during the late secretion stage and appear in the endometrial tissue. These processes can generate plasma cell-like interfering cells in the tissue that impede the diagnosis of chronic endometritis [9], thus causing high misdiagnosis and missed diagnosis rates.

Transmembrane heparin sulfate proteoglycan syndecan1 (CD138) is the most specific indicator of plasma cells. Specific staining of CD138 with immunohistochemical methods and direct detection of the existence of plasma cells can eliminate the interference of cells with similar morphology, and these methods are expected to improve the accuracy of chronic endometritis diagnosis. In the present study, HE staining was performed on endometrial tissues of 93 patients who underwent assisted pregnancy treatment. Of these cases, no patients were diagnosed with chronic endometritis, 25 patients were diagnosed with suspected chronic endometritis, and 68 patients were diagnosed with non-chronic endometritis. Whereas results from CD138 immunohistochemical staining showed that the positive rate of CD138 immunohistochemical staining of the 25 suspected CE patients was $12 \%$, the CD138 positive rate of endometrial tissue of the 68 non-chronic endometritis patients was $33.8 \%$, and the difference was statistically significant. This result suggests that even if pathology is the gold standard for the diagnosis of chronic endometritis, due to the difficulty of identification of plasma cells, conventional pathological diagnosis has a large degree of subjectivity, resulting in cases of misdiagnosis and missed diagnosis. This is consistent with the results from Bayer-Garner et al. In chronic endometritis, the plasma cell membrane shows strong positive CD138 immunohistochemical staining, while the cytoplasm shows weak positive staining, making the cells easy to discriminate in 200x and 400x magnification fields. Thus, in suspected chronic endometritis specimens, when plasma cells cannot be identified using HE staining, CD138 immunohistochemical staining can effectively display plasma cells, making plasma cells easy to identify, thereby improving the diagnosis rate of chronic endometritis. Therefore, CD138 immunohistochemical staining can improve the diagnosis rate and diagnostic accuracy of chronic endometritis [11].

The etiology of chronic endometritis is still unclear. Previous studies have suggested that chronic endometritis may be associated with bacterial infection, intrauterine devices, intrauterine abnormalities such as endometrial polyps, endometrial ossification, assisted adjuvant radiotherapy for endometrium cancer, and endometrial lymphoid intraepithelial neoplasia-like cancer [1]. The present study suggests that a previous prolonged menstrual bleeding episode history, an abortion history or fallopian tube obstruction are associated with the pathogenesis of chronic endometritis and are independent risk factors of chronic endometritis. Therefore, for infertile patients with the above mentioned risk factors, and especially for patients who undergo assisted conception treatment, a pathological endometrial CD138 immunohistochemical detection is recommended. Thus, a clear diagnosis and timely treatment can reduce the failure rate of assisted conception and improve the reproduction prognosis of patients.

In summary, on the basis of conventional endometrial HE staining and the conventional pathological examination, the addition of CD138 immunohistochemical staining can effectively improve the morphology examination and prevent missed diagnosis and misdiagnosis of some atypical cases of CE. Patients with a previous history of prolonged menstrual bleeding episodes, a history of abortion or complications of fallopian tube obstruction may be high-risk patients for chronic endometritis. These patients should be advised to have a CD138 immunohistochemical staining examination to clarify the diagnosis and undergo timely treatment to improve their reproductive prognosis. However, multi-center, large sample-size, evidence-based medical confirmation is still lacking, and the value of CD138 immunohistochemical staining still requires further clinical studies for verification.

\section{Conclusions}

CD138 immunohistochemistry can improve the CE diagnosis rate. A previous history of prolonged menstrual bleeding episodes, an abortion history, and a history of fallopian tube obstruction are risk factors for chronic endometritis, and a CD138 immunohistochemical examination should be advised among them.

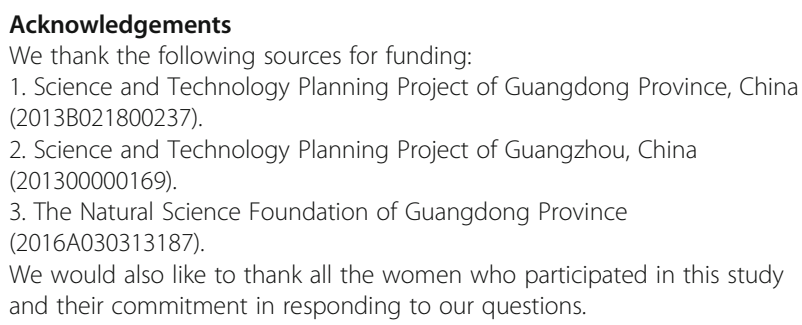


immunoassays. RF and $\mathrm{YL}$ participated in the acquisition and analysis of the data. RF participated in drafting the manuscript. YC and RF participated in revising the manuscript. All authors read and approved the final manuscript.

\section{Competing interests}

The authors declare that they have no competing interests.

\section{Consent for publication}

Not applicable.

\section{Ethics approval and consent to participate}

The present study has been reviewed and approved by the Ethics Committee ([2014] 64) of the First Affiliated Hospital of Sun Yat-sen University, and an informed consent was signed on by the patients involved.

\section{Author details}

'Department of Gynecology and Obstetrics, First Affiliated Hospital, Sun Yat-sen University, Guangzhou, Guangdong Province, People's Republic of China. ${ }^{2}$ Department of Gynecology and Obstetrics, Shenzhen Luohu People's Hospital, Shenzhen, Guangdong Province, People's Republic of China. ${ }^{3}$ Medical Records and Statistics Room, First Affiliated Hospital, Sun Yat-sen University, Guangzhou, Guangdong Province, People's Republic of China. ${ }^{4}$ Department of Pathology, First Affiliated Hospital, Sun Yat-sen University, Guangzhou, Guangdong Province, People's Republic of China.

Received: 7 May 2015 Accepted: 2 September 2016

\section{Published online: 05 September 2016}

\section{References}

1. Kasius JC, Fatemi HM, Bourgain C, Sie-Go DM, Eijkemans RJ, Fauser BC, Devroey P, Broekmans FJ. The impact of chronic endometritis on reproductive outcome. Fertil Steril. 2011;96(6):1451-6.

2. Carvalho FM, Aguiar FN, Tomioka R, de Oliveira RM, Frantz N, Ueno J. Functional endometrial polyps in infertile asymptomatic patients: a possible evolution of vascular changes secondary to endometritis. Eur J Obstet Gynecol Reprod Biol. 2013;170(1):152-6.

3. Polisseni F, Bambirra EA, Camargos AF. Detection of chronic endometritis by diagnostic hysteroscopy in asymptomatic infertile patients. Gynecol Obstet Invest. 2003;55(4):205-10.

4. Kitaya K. Prevalence of chronic endometritis in recurrent miscarriages. Fertil Steril. 2011;95(3):1156-8.

5. Bouet PE, El HH, Monceau E, Gariepy G, Kadoch IJ, Sylvestre C. Chronic endometritis in women with recurrent pregnancy loss and recurrent implantation failure: prevalence and role of office hysteroscopy and immunohistochemistry in diagnosis. Fertil Steril. 2016;105(1):106-10.

6. Johnston-MacAnanny EB, Hartnett J, Engmann LL, Nulsen JC, Sanders MM, Benadiva CA. Chronic endometritis is a frequent finding in women with recurrent implantation failure after in vitro fertilization. Fertil Steril. 2010; 93(2):437-41.

7. Cicinelli $E$, Matteo $M$, Tinelli $R$, Lepera A, Alfonso R, Indraccolo U, Marrocchella S, Greco P, Resta L. Prevalence of chronic endometritis in repeated unexplained implantation failure and the IVF success rate after antibiotic therapy. Hum Reprod. 2015;30(2):323-30.

8. Tortorella C, Piazzolla G, Matteo M, Pinto V, Tinelli R, Sabba C, Fanelli M, Cicinelli E. Interleukin-6, interleukin-1 beta, and tumor necrosis factor alpha in menstrual effluents as biomarkers of chronic endometritis. Fertil Steril. 2014;101(1):242-7.

9. Greenwood SM, Moran JJ. Chronic endometritis: morphologic and clinical observations. Obstet Gynecol. 1981:58(2):176-84.

10. Adegboyega PA, Pei Y, McLarty J. Relationship between eosinophils and chronic endometritis. Hum Pathol. 2010:41(1):33-7.

11. Bayer-Garner IB, Nickell JA, Korourian S. Routine syndecan-1 immunohistochemistry aids in the diagnosis of chronic endometritis. Arch Pathol Lab Med. 2004;128(9):1000-3.

12. Kiviat NB, Wolner-Hanssen P, Eschenbach DA, Wasserheit JN, Paavonen JA, Bell TA, Critchlow CW, Stamm WE, Moore DE, Holmes KK. Endometrial histopathology in patients with culture-proved upper genital tract infection and laparoscopically diagnosed acute salpingitis. Am J Surg Pathol. 1990;14(2):167-75.

13. Maleki Z, Bailis AJ, Argani CH, Askin FB, Graham EM. Periventricular leukomalacia and placental histopathologic abnormalities. Obstet Gynecol. 2009;114(5):1115-20.

\section{Submit your next manuscript to BioMed Central and we will help you at every step:}

- We accept pre-submission inquiries

- Our selector tool helps you to find the most relevant journal

- We provide round the clock customer support

- Convenient online submission

- Thorough peer review

- Inclusion in PubMed and all major indexing services

- Maximum visibility for your research

Submit your manuscript at www.biomedcentral.com/submit
Biomed Central 